\title{
The effects of incremental whole beetroot consumption on plasma nitrate and nitrite levels and blood pressure in young and old subjects
}

\author{
Tess E Capper ${ }^{1,2}$, Daniel J West ${ }^{1,2}$, Mario Siervo ${ }^{1,2}$ and Emma J Stevenson ${ }^{1,2}$ \\ ${ }^{1}$ Institute of Cellular Medicine, Newcastle University, NE1 $4 H H$ and \\ ${ }^{2}$ Human Nutrition Research Centre, Newcastle University, NE1 $4 \mathrm{HH}$.
}

Nitric oxide (NO) is produced in the body endogenously or through the breakdown of exogenous dietary nitrate, and has been found to reduce blood pressure (BP) and improve endothelial function ${ }^{1,2}$. The richest sources of dietary nitrate come from vegetables such as radish, rocket and beetroot, the latter of which has been supplemented in a variety of forms, most commonly as a juice. This study investigated the effects of whole beetroot on BP following incremental doses in a young and old population, and compared the effects to a positive control. We hypothesised that there would be a nitrate dose-dependent response in BP, and that the old would have a reduced response to these doses in comparison with the young. Additionally, we hypothesised that there would be a direct association between changes in BP and plasma nitrate and nitrite concentrations. In this randomised, crossover trial, 12 adults aged 18-35 and 12 adults aged 60-75 consumed either $100 \mathrm{~g}, 200 \mathrm{~g}$, or $300 \mathrm{~g}$ whole beetroot or a $1 \mathrm{~g}$ potassium nitrate solution (PN) on four separate occasions, and BP was assessed over a five-hour period following consumption. Results showed that for systolic BP, there was no significant effect of trial, but a significant effect of time $(p=0.034)$ and a time*age interaction $(p=0.013)$. There was a significant effect of time $(p<0.001)$ and a significant age effect on diastolic BP $(p=0.025)$ (Figure 1). Results also show that there was a dosedependent increase in nitrate and nitrite plasma concentrations and greater increases in plasma nitrite concentrations were associated with a greater decrease in both systolic $(n=288, r=-0.28, p<0.001)$ and diastolic $(n=288, r=-0.25, p<0.001)$ blood pressure.

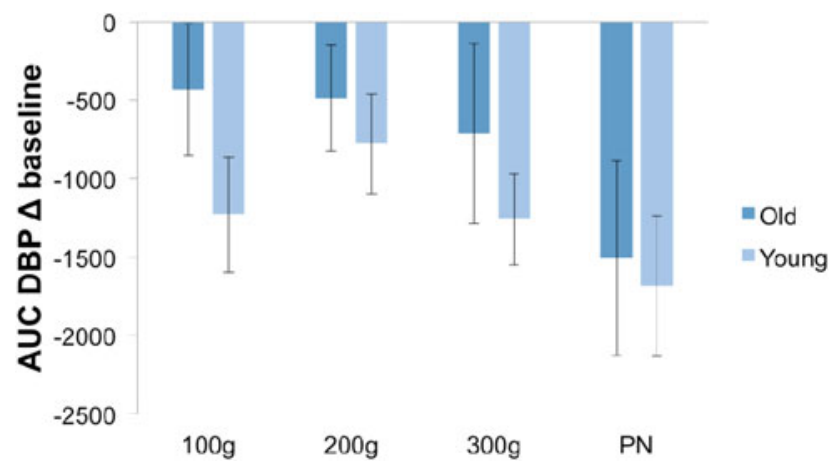

Fig. 1. Area under the curve (AUC) of change in diastolic blood pressure (DBP) from baseline in the young and old groups. Data presented as mean \pm $\mathrm{SEM}, \mathrm{n}=24$ (12 young and 12 old $)$.

Results demonstrate that consumption of beetroot in its whole form can increase plasma concentrations of nitrate and nitrite, which in turn can exert positive effects on the vascular system in both young and old adults. Higher doses of nitrate are required to elicit comparable effects in an older population, which can be achieved through consumption of larger amouts of whole beetroot. Future work is warranted into the mechanisms behind response differences in young and older adults and a lower sensitivity to the beneficial effects of dietary nitrate in older age. This may have implications for healthy cardiovascular ageing.

\section{References}

1. Asgary S. et al. (2016) 'Improvement of hypertension, endothelial function and systemic inflammation following short-term supplementation with red beet (Beta vulgaris L.) juice: A randomized crossover pilot study', Journal of Human Hypertension, 30(10), pp. 627-632.

2. Larsen F. J. et al. (2006) 'Effects of dietary nitrate on blood pressure in healthy volunteers', The New England journal of medicine, 355(26), pp. 27922793. 